\title{
Mitochondrial TERT enhances mitochondria functions in vivo by protecting mitochondrial DNA integrity from oxidative damage N Buechner*1, S Droese ${ }^{2}$, S Jakob ${ }^{1}$, U Brandt ${ }^{2}$, J Altschmied ${ }^{1}$ and J Haendeler ${ }^{1}$
}

\author{
Address: ${ }^{1}$ Institut für umweltmedizinische Forschung, molekulare Alternsforschung, AG Haendeler, Düsseldorf, Germany and ${ }^{2}$ Insitut für \\ Bioenergetik, Universität Frankfurt, Frankfurt, Germany \\ * Corresponding author
}

from I2th Joint Meeting of the Signal Transduction Society (STS). Signal Transduction: Receptors, Mediators and Genes

Weimar, Germany. 29-3I October 2008

Published: 26 February 2009

Cell Communication and Signaling 2009, 7(Suppl I):A57 doi:10.II86/1478-8IIX-7-SI-A57

This abstract is available from: http://www.biosignaling.com/content/7/SI/A57

(c) 2009 Buechner et al; licensee BioMed Central Ltd.

Mitochondria are essential for respiration and oxidative phosphorylation. Mitochondrial dysfunction due to aging processes is involved in pathologies and pathogenesis of a series of cardiovascular disorders. New results accumulate showing that the enzyme telomerase with its catalytic subunit telomerase reverse transcriptase (TERT) has a beneficial effect on heart functions. The benefit of short-term running of mice for heart function is dependent on TERT expression. TERT can translocate into the mitochondria and mitochondrial TERT (mtTERT) is protective against stress induced stimuli and binds to mitochondrial DNA (mtDNA). Because mtDNA is highly susceptible to damage produced by reactive oxygen species (ROS) which are generated in close proximity to the respiratory chain, the aim of this study was to determine the functions of mtTERT in vivo and in vitro. Therefore, mitochondria from hearts of adult, 2nd generation TERT-deficient mice (TERT $-/-)$ and wt littermates were isolated and state 3 respiration was measured. Strikingly mitochondria from TERT -/revealed a significantly lower state 3 respiration (TERTwt: $987+/-72 \mathrm{pmol} / \mathrm{s}^{*} \mathrm{mg}$ vs. TERT-/-: $774+/-38 \mathrm{pmol} / \mathrm{s}^{*} \mathrm{mg}$, $\mathrm{p}<0.05, \mathrm{n}=5$ ). These results demonstrated that TERT $-/-$ mice have a so far undiscovered heart phenotype. In contrast mitochondria isolated from liver tissues did not show any differences. To get further insights in the molecular mechanisms, we reduced endogenous TERT levels by shRNA and measured mitochondrial reactive oxygen species (mtROS). mtROS were increased after ablation of TERT (scrambled: $4.98+/-1.1 \%$ gated vs. shTERT: $2.03+$ +/ - $0.7 \%$ gated, $\mathrm{p}<0.05, \mathrm{n}=4)$. We next determined mtDNA deletions, which are caused by mtROS. Semi- quantitative realtime PCR of mtDNA deletions revealed that mtTERT protects mtDNA from oxidative damage. To analyze whether mitochondrial integrity is required to protect from apoptosis, vectors with mitochondrially targeted TERT (mitoTERT) and wildtype TERT (wtTERT) were transfected and apoptosis was measured. mitoTERT showed the most prominent protective effect on $\mathrm{H}_{2} \mathrm{O} 2$ induced apoptosis. In conclusion, mtTERT has a protective role in mitochondria by importantly contributing to mtDNA integrity and thereby enhancing respiration capacity of the heart. 